Original Research

\title{
Consumption of a Recommended Serving of Wheat Bran Cereals Significantly Increases Human Faecal Butyrate Levels in Healthy Volunteers and Reduces Markers of Inflammation Ex Vivo
}

Madalina Neacsu ${ }^{1,}{ }^{*}$, Susan E. Anderson ${ }^{2}$, Pola Verschoor ${ }^{1}$, Nicholas J. Vaughan ${ }^{1}$, Graham W. Horgan ${ }^{3}$, Toine Hulshof ${ }^{4}$, Sylvia H. Duncan ${ }^{1}$, Susan Duthie ${ }^{1,5}$, Wendy R. Russell ${ }^{1}$

1. Gut Heath, University of Aberdeen Rowett Institute, Aberdeen AB25 2ZD, UK; E-Mails: m.neacsu@abdn.ac.uk; pola.verschoor@abdn.ac.uk; nick.vaughan@abdn.ac.uk; sylvia.duncan@abdn.ac.uk; s.j.duthie@rgu.ac.uk; w.russell@abdn.ac.uk

2. Scientific Services, University of Aberdeen Rowett Institute, Aberdeen AB25 2ZD, UK; E-Mail: s.anderson@abdn.ac.uk

3. Biomathematics and Statistics Scotland, Aberdeen AB25 2ZD, Scotland, UK; E-Mail: g.horgan@abdn.ac.uk

4. Kellogg's Europe, Orange Tower, Media City UK, Salford, Manchester M50 2HF, UK; E-Mail: toine.hulshof@kellogg.com

5. School of Pharmacy and Life Sciences, Robert Gordon University, Aberdeen, UK

* Correspondence: Madalina Neacsu; E-Mail: m.neacsu@abdn.ac.uk

Academic Editor: Jennifer Keogh

Special Issue: Feature Papers of Recent Progress in Nutrition 2021

Recent Progress in Nutrition

2021, volume 1 , issue 4

doi:10.21926/rpn.2104002
Received: October 08, 2021

Accepted: December 13, 2021

Published: December 17, 2021

\section{Abstract}

Wheat bran cereals are an important source of dietary fibre. The aim of the study was to investigate if a high intake (120 g) of fibre rich breakfast cereal (which delivers the UK Government guidelines for fibre intake in one serving but is three-fold higher than the manufacturers recommended serving) has additional potential health benefits compared to the recommended serving ( $40 \mathrm{~g}$, containing $11 \mathrm{~g}$ of dietary fibre). To assess this, the study determined the short chain fatty acid (SCFA) profiles in human faecal, urine and plasma

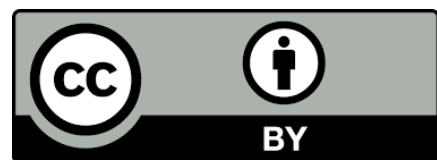


samples after consumption of two different servings of fibre-rich cereal. Inhibition of prostanoid production was measured (ex vivo) in human colonic fibroblast cells after cytokine (IL-1ß) inflammation stimulation. Eight healthy volunteers, $18-55$ years old; BMI $\left(18-30 \mathrm{~kg} / \mathrm{m}^{2}\right)$ consumed the wheat bran-rich "ready to eat cereal", at both the high (120 g) serving and recommended (40 g) serving. Faecal, urine and plasma samples were collected at baseline, throughout the five-hour intervention period and approximately 24 hours following consumption. Faecal butyrate showed the largest increase $(p<0.05)$ of more than a two-fold change following the consumption of the recommended serving of wheat bran cereal (from $13.95 \pm 9.17$ to $31.63 \pm 20.53 \mathrm{mM}$ ) and no significant change following the higher serving (from $21.96 \pm 11.03$ to $22.9 \pm 12.69 \mathrm{mM}$ ). ANOVA analysis also found a weak serving effect ( $p$ $=0.046$ ) of the portion size (high vs. recommended) only for butyrate in urine 24 hours after consumption of the bran cereal. The physiological nutritionally relevant concentrations of faecal SCFAs, as determined in the volunteers' faecal samples showed significant antiinflammatory activity or the individual faecal SCFAs; acetate $(p<0.001)$, propionate $(p<0.001)$ and butyrate $(p<0.01)$, as well as in combination. Plasma folate was also increased after consumption of both wheat bran servings and was significant $(p=0.037)$ at the three-hour time point following consumption of the high wheat bran serving. The consumption of the recommended serving ( $40 \mathrm{~g}$ ) of wheat bran cereal increased the total microbial SCFAs levels (from 96.88 to $136.96 \mathrm{mM}$ ) compared to the higher serving ( $120 \mathrm{~g}$ ) (from 110.5 to $117.64 \mathrm{mM}$ ) suggesting that the intake of the higher portion size is likely to promote a faecal bulking effect and thereby decrease colonic SCFA levels. These data indicate that consumption of the recommended serving of wheat bran cereal serving would therefore be sufficient to promote microbial butyrate formation, reduce colonic inflammation and increase plasma folate levels in humans.

\section{Keywords}

Butyrate; dietary fibre; gut inflammation; short chain fatty acids; wheat bran cereals; folate

\section{Introduction}

In 2015, as part of the initiative to promote a healthy balanced diet, the UK government changed the guidelines on the daily recommended dietary fibre intake to $30 \mathrm{~g}$ per day [1], representing $23 \mathrm{~g}$ of non-starch polysaccharides (NSP), a 5 g increase over the previous $18 \mathrm{~g}$ NSP recommendation [2]. This dietary fibre value ( $30 \mathrm{~g} /$ day) refers to AOAC fibre, meaning measured by the Association of Official Analytical Chemists' (AOAC) method [2]. However, most adults in the UK are only eating an average of approx. $18 \mathrm{~g}$ dietary fibre per day [1]. The UK data for adults [3] shows that cereal products contribute $37 \%$ of fibre intake and $28 \%$ of total calorie intake and that wheat consumption is more than ten-times that of rice, oats, or maize [4]. Bran fractions produced by milling are nutritionally rich in minerals, vitamin B6, thiamine, folate and vitamin $E$, as well as phenolic compounds [5]. One of the richest sources of fibre from wheat bran-based products is breakfast cereals [4]. Furthermore, breakfast cereals are also an important source of folate in the human diet. Folate has a key role in ensuring normal development, growth, and maintenance of optimal health 
[6] and maintaining an optimal folate status in the population is a public health priority, in 2021, the UK Government adopted mandatory folic acid fortification, and folate will be added to nonwholemeal wheat flour across the UK to help prevent life-threatening spinal conditions in babies [7]. Metabolic changes associated with impaired folate status have been linked to increased risk of chronic diseases including cancer, cardiovascular disease and cognitive dysfunction [8]. The role of folic acid in gut heath is still a subject of debate [9].

Amongst other suggested mechanisms by which wheat bran could benefit gut health [10] is the production of microbially-produced short chain fatty acids (SCFA) mainly produced by the dense population of bacteria in the large intestine that ferment complex carbohydrates that escape digestion by host enzymes [11]. Much of the fermentation of these carbohydrates occurs in the proximal colon, with reduced levels of carbohydrates available for fermentation by bacteria in the distal colon which is the main site of polyp development [12]. These SCFA and in particular butyrate [13] are the major energy source for colonocytes and may modulate carcinogenesis through their effects on proliferation, differentiation and apoptosis in the gut, as well as stimulation of the immune system [14]. Many of the inflammatory pathways regulating the production of prostanoids are implicated in the development of colon cancer. Consequently, chemo-preventive strategies to inhibit development and induce regression of colorectal adenomas often involve modulation of the pathways regulating prostaglandin production [15].

Meeting the dietary fibre daily recommendation is on the agenda of many countries, as globally only a few countries meet or exceed this amount. Little is known regarding the best consumption pattern to follow to achieve optimal health benefits from fibre consumption. The aim of this study was to investigate the potential health benefits of a three-fold higher intake of recommended (by manufacturer) serving size of wheat bran cereal $(120 \mathrm{~g})$, representing the approximate UK Government guidelines daily recommendation for fibre (33 g), compared with the manufacturer recommended serving ( $40 \mathrm{~g}$ ) consumption in one meal, representing approximate a third of the daily fibre recommendation ( $11 \mathrm{~g}$ ). The study measured the concentrations of SCFAs faecal, urine and plasma samples and plasma folate following the consumption of two different servings of wheat bran cereals by healthy human volunteers. The study also determined the impact of physiologically relevant in vivo faecal SCFA concentrations, mixtures and ratios measured in healthy volunteers following the consumption of wheat bran cereals on inhibition of prostanoid production using colonic fibroblast cells (ex vivo). The outcome of this study suggests that a recommended $40 \mathrm{~g}$ serving of bran cereal results in increased levels of faecal butyrate which is likely to be health protective.

\section{Materials and Methods}

\subsection{Human Study}

\subsubsection{Study Design}

Details of the human dietary intervention study were published previously [16]. Briefly, eight healthy volunteers (seven females, one male), $38.62 \pm 10.8$ years old; $23.39 \pm 2.63 \mathrm{~kg} / \mathrm{m}^{2}$ (BMI), were recruited for a non-randomised, cross-over acute human dietary intervention study. Written informed consent was obtained from each subject. Each volunteer consumed wheat bran-rich cereals, a high (120 g) serving with semi-skimmed milk (375 mL)- HWB (high wheat bran and a $40 \mathrm{~g}$ 
serving with semi-skimmed milk ( $125 \mathrm{~mL}$ )- WB (manufacturer recommended portion of wheat bran), following a three-day low phytochemical and fibre diet. No food and drinks (apart from water) were consumed by the volunteers until the completion of collection of $5 \mathrm{~h}$ blood and urine samples. There was a washout period of at least two weeks between interventions. Volunteers were fasted for 12 $\mathrm{h}$ prior to commencing the intervention and were requested not to drink or eat anything within the first $5 \mathrm{~h}$ following consumption. For the remaining intervention period $(24 \mathrm{~h})$, volunteers could consume the low phytochemical diet. Faecal, urine and blood samples were collected prior to the beginning of each intervention and at 1, 3, 5 and 24 hours (for bloods and urine) and first faecal sample after consuming the cereals, labelled as $24 \mathrm{~h}$ for simplicity (Figure 1 ).
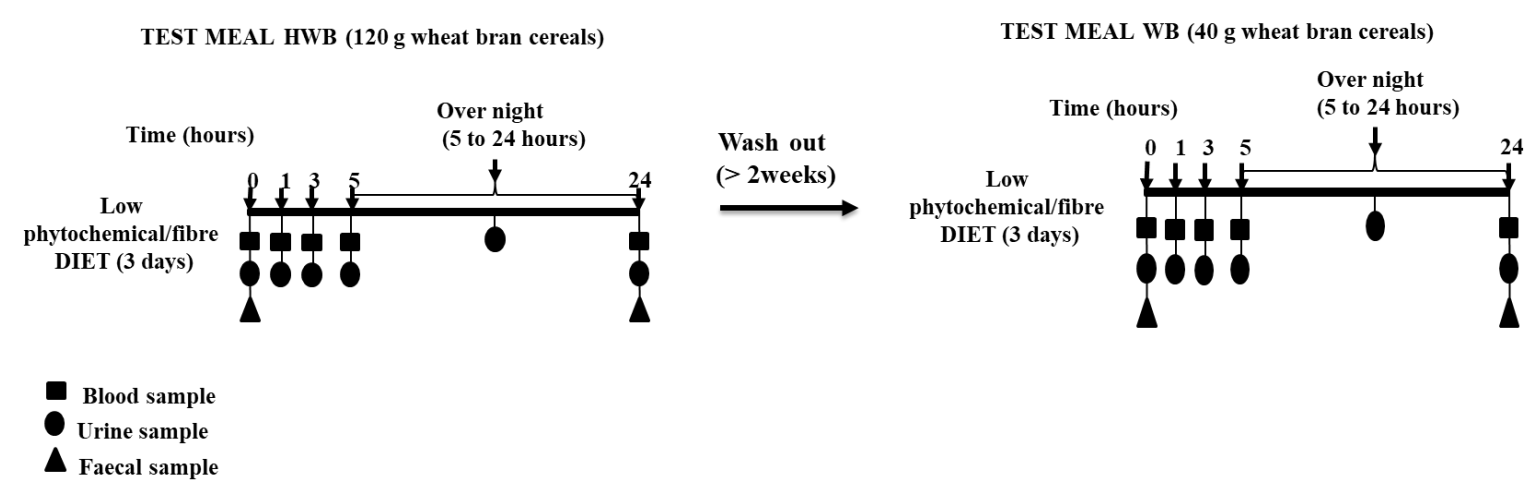

Figure 1 Human dietary intervention study diagram: Each volunteer $(n=8)$ consumed wheat bran-rich cereals at three times the manufacturer's recommended serving (120 g) with semi-skimmed milk ( $375 \mathrm{~mL}$ ) - HWB (High Wheat Bran intervention day) and one recommended serving ( $40 \mathrm{~g}$ ) along with semi-skimmed milk (125 mL)-WB (Wheat Bran Intervention day) following a 3-day low phytochemical and fibre diet with a washout period of at least two weeks between intervention days. Sample collection: prior test meal, baseline $(0 \mathrm{~h})$ and first faecal sample after wheat bran consumption (approx. $24 \mathrm{~h}$ after); baseline ( $0 \mathrm{~h}), 1,3,5$ and $24 \mathrm{~h}$ blood; and baseline $(0 \mathrm{~h}), 1,3,5$, overnight (from $5 \mathrm{~h}$ until $24 \mathrm{~h}$ ) and $24 \mathrm{~h}$ urine.

\subsubsection{Intervention Product}

The study used All Bran original cereal (Kellogg's, UK). According to the manufacturer, one recommended serving $(40 \mathrm{~g}$ ) of the product with $125 \mathrm{ml}$ milk contain $10 \mathrm{~g}$ protein, $25 \mathrm{~g}$ carbohydrate (of which $13 \mathrm{~g}$ sugars, $12 \mathrm{~g}$ starch), $3.5 \mathrm{~g}$ fat (of which $1.5 \mathrm{~g}$ saturated fat), $11 \mathrm{~g}$ fibre and $0.8 \mathrm{~g}$ salt. According to the manufacturer one recommended serving of wheat bran cereals ( $40 \mathrm{~g}$ ) contains 108 $\mu \mathrm{g}$ folic acid, providing $54 \%$ of the folate RDA.

\subsubsection{Human Sample Processing}

The faecal, urine and blood samples were processed as previously described [16] and stored at $80^{\circ} \mathrm{C}$ until SCFA and plasma folate analysis was performed. Briefly, blood samples were collected directly into heparinised tubes at the $0,1,3,5$ and $24 \mathrm{~h}$ time points. The samples were centrifuged $\left(1500 \times \mathrm{g}, 15 \mathrm{~min} ; 4^{\circ} \mathrm{C}\right)$ within $45 \mathrm{~min}$ to separate the plasma. Urine samples were measured by weight and volume at the same time points up to $5 \mathrm{~h}$, and then the total $24-\mathrm{h}$ sample collected. The 
harvested plasma and the urine were aliquoted and stored at $-80^{\circ} \mathrm{C}$. The faecal samples were weighed, mixed and immediately frozen at $-80^{\circ} \mathrm{C}$. Faecal samples were thawed, mixed once again, weighed and the faecal waters separated using a high-speed centrifuge (50 $000 \times \mathrm{g} ; 2 \mathrm{~h} ; 10^{\circ} \mathrm{C}$ ). The supernatant (faecal water) was carefully removed, aliquoted into Eppendorf tubes and immediately stored at $-80^{\circ} \mathrm{C}$ for analyses.

\subsection{Human Samples Analysis}

\subsubsection{Analysis of SCFAs in Urine, Plasma and Faecal Samples}

Acetate, propionate and butyrate were analysed by GC-MS as previously described [17] following derivatisation of the samples using $\mathrm{N}$-tert-butyldimethylsilyl- $\mathrm{N}$-methyltrifluoroacetamide (MTBSTFA). To an accurately weighed ( $0.4 \mathrm{~g}$ ) plasma and urine sample $0.025 \mathrm{~g}$ of internal standard (13C isotopes of acetic, propionic, and butyric) and approximately $10 \mathrm{mg}$ of 5-sulphosalicylic acid was added. The internal standards for acetic, propionic and butyric were added to plasma and urine samples and proceed the extraction and derivatisation for GC-MS analysis using MTBSTFA (30 \%) in acetonitrile. For the analysis of acetic, propionic and butyric acids from the faecal samples, $10 \mu \mathrm{L}$ of faecal water was added to $1.855 \mathrm{~mL}$ of water, then $200 \mu \mathrm{L}$ was transferred and prepared for analysis as described above. The samples were analysed using an Agilent 5973 MSD GC-MS. Separation was achieved using an Alltech EC1 capillary column $30 \mathrm{~m} \times 0.25 \mathrm{~mm}$ ID $\times 0.25 \mu \mathrm{m}$ film thickness. Samples were injected $(1 \mu \mathrm{L})$ in split mode 40:1. Helium was used as the carrier gas. The injector temperature

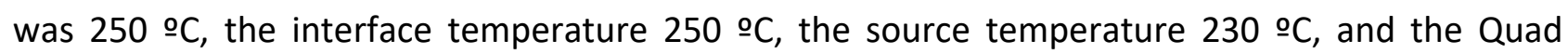
temperature $200 \stackrel{\circ}{ } \mathrm{C}$. Ions monitored (areas obtained) for labelled and unlabelled SCFA were as follows $(M+1 / M+$ apart from butyric $M+2 / M+)$ : acetic, $117.1 \& 118.1$; propionic, 131.1 \& 132.1 ; and butyric $145.2 \& 147.2$.

\subsubsection{Analysis of Plasma Folates}

Plasma folate was measured using a radioactive competitive binding assay SimulTRAC-SNB Radioassay Kit Vitamin B12 [ ${ }^{57}$ Co]/Folate [ ${ }^{125}$ I], ICN Diagnostics, Orangeburg, NY.

\subsection{Cell Culture}

Human colonic fibroblast cells CCD-18Co (CRL-1459) were obtained from the American Type Culture Collection Middlesex, England. Cells were purchased at a population doubling (PDL) of 27, and all studies were performed on cells from the fifth passage (PDL 30.7). The number of viable cells was measured using a colorimetric assay CellTiter96\AQueous; Promega; Madison, WI.

\subsection{Anti-inflammatory Screening Assay Cell Culture}

The ability of the SCFAs to inhibit neoplastic prostanoid production in colon cells following an inflammatory insult was performed as described previously [15]. The fibroblast cells (passage 5; PDL 30.7) were seeded at a density $1 \times 10^{4}$ well-1. The cells were treated with acetate $(73.57 \mathrm{mM})$, propionate $(26.46 \mathrm{mM})$, and butyrate $(27.27 \mathrm{mM})$ and their mixture respectively as mean concentrations, determined in the faecal water samples after the consumption of wheat bran servings, thirty minutes prior to stimulation with IL-1ß. Prostaglandin concentrations were 
measured using a prostaglandin screening EIA kit (Cayman Chemicals; Ann Arbor, MI) designed to measure a wide range of prostanoids.

\subsection{Statistical Analysis}

All data are expressed as mean \pm standard deviation (STD). The effect of treatment on SCFA was assessed by analysis of variance, with each time point considered a distinct outcome, with terms for volunteer, treatment and baseline value. There were some indications of skewed distributions, so analysis was repeated on a log scale to confirm conclusions. The cell culture data and folate levels were analysed by paired t-tests to examine the differences between treatments and concentrations of SCFA used.

\subsection{Ethics Statement}

The study followed all the principles of the Declaration of Helsinki and good clinical practice, and all procedures involving human subjects were reviewed and approved by the Rowett Institute Ethics Panel, Study Number 2001, University of Aberdeen, UK. The study was registered with clinicaltrial.gov, study ID number: NCT02177279.

\section{Results}

\subsection{SCFA Content of Human Faecal Waters, Plasma, and Urine}

Faecal waters contained the highest concentrations of SCFA (Table 1) compared to that of plasma (Figure 2A) and urine samples (Figure 2B). The highest increase in faecal SCFA concentration when compared with baseline values was following consumption of WB serving for butyrate (more than two-fold increase) Table 1. The overall mean concentrations of SCFA in the faecal water samples following the consumption of both WB and HWB (for $n=8$ volunteers) were $73.57 \pm 30.77 \mathrm{mM}$ for acetate; $26.46 \pm 11.45 \mathrm{mM}$ for propionate and $27.27 \pm 17.09 \mathrm{mM}$ for butyrate. Samples collected following the intervention showed no significant changes in faecal samples weights and total faecal waters produced between baseline and first sample produced following HWB consumption ( $p>0.05$, $t$ test) and WB consumption ( $p>0.05, t$ test) (Table $S 1$, supplementary information).

Table 1 SCFA faecal water concentrations (mean \pm STD; $n=8$ ) and $\%$ change between baseline and post intervention following wheat bran (WB) and high wheat bran (HWB) consumption.

\begin{tabular}{|c|c|c|c|c|c|c|}
\hline \multirow[b]{2}{*}{ SCFA } & \multicolumn{3}{|c|}{ HWB (120 g) } & \multicolumn{3}{|c|}{ WB (40 g) } \\
\hline & Baseline & $\begin{array}{l}\text { Post } \\
\text { Intervention }\end{array}$ & $\begin{array}{l}\text { Change } \\
(\%)\end{array}$ & Baseline & $\begin{array}{l}\text { Post } \\
\text { Intervention }\end{array}$ & $\begin{array}{l}\text { Change } \\
(\%)\end{array}$ \\
\hline Acetate & $65.59 \pm 16.9$ & $69.19 \pm 24.26$ & +5.48 & $60.62 \pm 15.86$ & $77.95 \pm 37.37$ & +28.58 \\
\hline Propionate & $22.68 \pm 10.03$ & $25.55 \pm 10.08$ & +12.65 & $22.31 \pm 9.76$ & $27.38 \pm 13.31$ & +22.75 \\
\hline Butyrate & $21.96 \pm 11.03$ & $22.9 \pm 12.69$ & +4.28 & $13.95 \pm 9.17$ & $31.63 \pm 20.53$ & $+126.78^{*}$ \\
\hline
\end{tabular}




\begin{tabular}{lcccccc}
$\begin{array}{l}\text { Total }(\mathrm{mM}) \\
\text { \% Butyrate }\end{array}$ & 110.23 & 117.64 & +6.72 & 96.88 & 136.96 & +39.54 \\
(from total) & 19.91 & 19.46 & -2.31 & 14.36 & 23.09 & +62.19 \\
\hline
\end{tabular}

Significant changes in SCFAs measured in faecal waters refer to the difference between time 0 (baseline) prior the consumption of the test meals and the post intervention sample after consumption of test meals, where ${ }^{*} p<0.05$, significant change between baseline and post intervention (t-test).

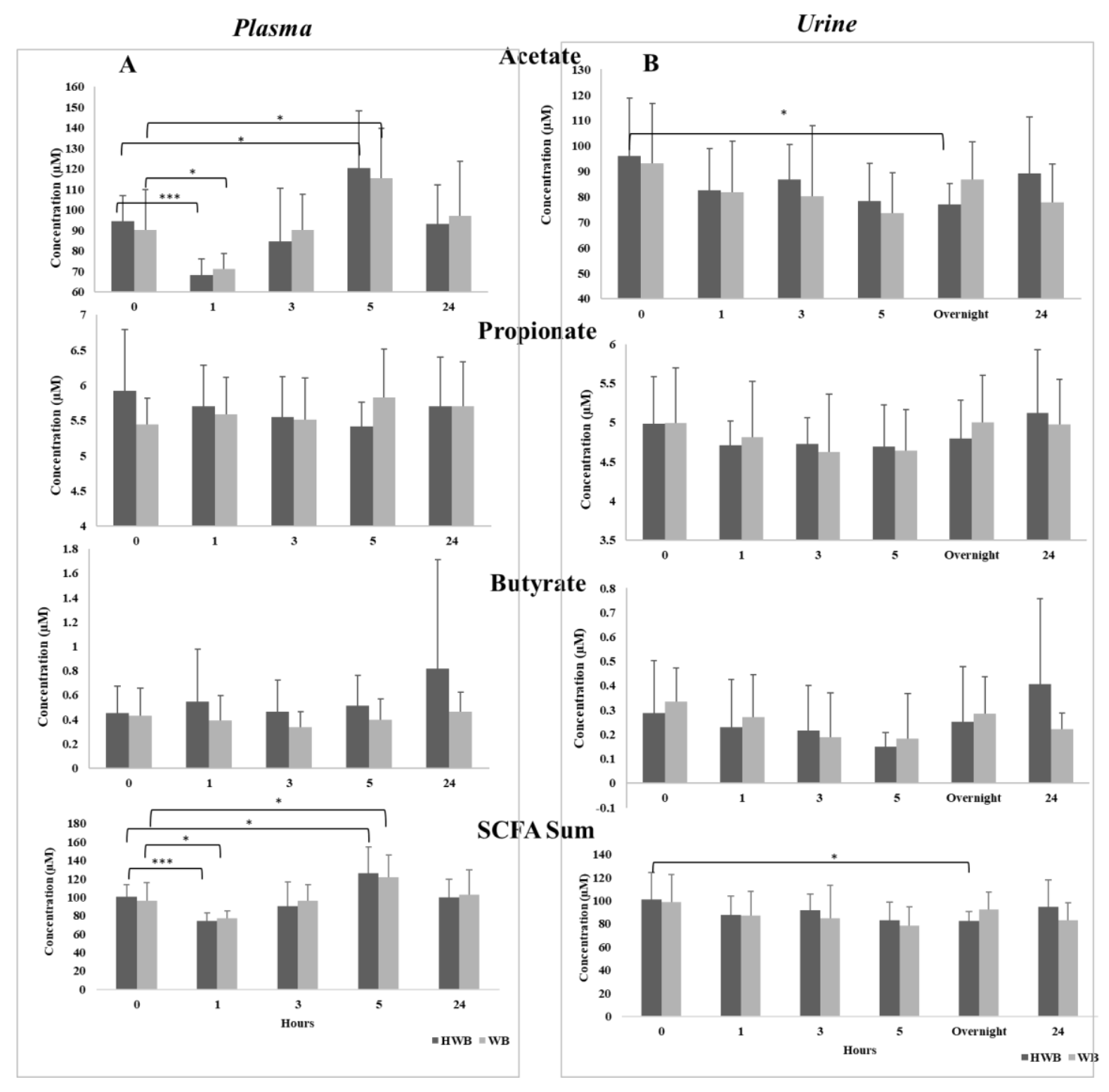

Figure 2 Plasma distribution (A) and urine distribution (B) of SCFA concentration (mean \pm STD, for $n=8$ volunteers) of acetate, propionate, butyrate and total SCFAs measured in human plasma samples at baseline ( $0 \mathrm{~h}$ ), and 1, 3, 5, $24 \mathrm{~h}$ and baseline (0h), and 1, 3, 5 , (overnight from $5 \mathrm{~h}$ to $24 \mathrm{~h}$, on plot $19 \mathrm{~h}$ ), $24 \mathrm{~h}$ (for urine samples) after consumption of HWB, (black) and respectively recommended serving of WB (grey). Where ${ }^{*}$ stands for $p<0.05$ and ${ }^{* * *}$ for $p<0.001$ for significant change vs baseline, when calculated with t-test. All the significance changes in SCFAs measured in biological samples refer to the difference between time 0 (baseline) prior the consumption of the test meals and time points after consumption of test meals. 
There were significant effects overall with time for propionate $(p<0.05)$, and with time $(p=0.01)$, and serving $(p<0.05)$ for butyrate. This translates to a significant increase $(p=0.024$, t-test) in butyrate concentration in faecal samples following WB consumption (from $13.95 \pm 9.17 \mathrm{mM}$ to $31.63 \pm 20.5 \mathrm{mM}$ ), representing a $127 \%$ increase. The results presented in this in vivo study are in accordance with previous research [18], where in vitro butyrate levels were also elevated when bran was fermented by mixed microbiota in studies conducted in model colonic fermenter systems.

In the plasma samples, acetate was the most concentrated SCFA found, with the highest levels determined at $5 \mathrm{~h}$ after consumption of WB and HWB $(115.51 \pm 24.07$ and $120.43 \pm 27.84 \mu \mathrm{M}$ respectively), followed by propionate and butyrate. ANOVA showed an overall significant effect $(p<0.001)$ for time on plasma concentrations for acetate (at $1 \mathrm{~h} p<0.001$, and $5 \mathrm{~h} p<0.01$; t-test). This translates to a significant decrease in plasma acetate concentration at 1 hour after HWB consumption (from $94.36 \pm 12.68 \mu \mathrm{M}$ to $68.32 \pm 7.87 \mu \mathrm{M}, \mathrm{n}=8 ; \mathrm{p}<0.001$, t-test) and at 1 hour after WB (from $90.19 \pm 19.71 \mu \mathrm{M}$ to $71.22 \pm 7.67 \mu \mathrm{M} ; \mathrm{p}<0.01$, t-test); and a significant increase in plasma acetate at 5 hours after WB consumption (from $90.19 \pm 19.71 \mu \mathrm{M}$ to $115.51 \pm 24.07 \mu \mathrm{M}$; $\mathrm{p}<0.05$, ttest).

The SCFA distribution pattern in urine was similar to plasma; for butyrate at $24 \mathrm{~h}$ where there was a weak serving effect $(p<0.05$, ANOVA). Overall, ANOVA showed a significant effect $(p<0.05)$ for time and butyrate concentration with t-test values of $p<0.05$ at $3 h ; p<0.01$ at $5 h$; and $p<0.05$ overnight. There was also a significant effect for time for acetate at $5 \mathrm{~h}$ and overnight $(p<0.05)$ in comparison with baseline. These translates to a significant decrease ( $p<0.05, t$-test) in butyrate concentration overnight for urine samples after WB consumption (from $0.33 \pm 0.14 \mu \mathrm{M}$ to $0.23 \pm$ $0.08 \mu \mathrm{M})$.

There was a significant decrease of plasma total SCFAs concentration at $1 \mathrm{~h}$ (vs baseline) following WB ( $p<0.05$, t-test) and HWB ( $p<0.001$, t-test) and a significant increase in 5 hours plasma following both $W B$ and HWB ( $p<0.05$, t-test) (Figure $2 A)$. Total SCFAs concentration were significantly reduced ( $p<0.05$, t-test) in overnight urine samples following HWB consumption (Figure 2B).

There were no differences between the wheat bran servings (HWB and WB) in plasma or urine concentrations of SCFAs.

\subsection{Folate Plasma Content}

Consumption of wheat bran led to an increase of plasma folate levels following both recommended and high servings WB (from $6.95 \pm 0.62$ to $8.17 \pm 0.43 \mathrm{ng} / \mathrm{mL}, \mathrm{n}=8$ in $5 \mathrm{~h}$ ) and HWB (from $6.77 \pm 0.62$ to $9.00 \pm 0.74 \mathrm{ng} / \mathrm{mL}, \mathrm{n}=8$ in $3 \mathrm{~h}$ ). This rise was significant ( $p<0.05$, t-test) only after consuming the HWB serving (Figure 3 ). 


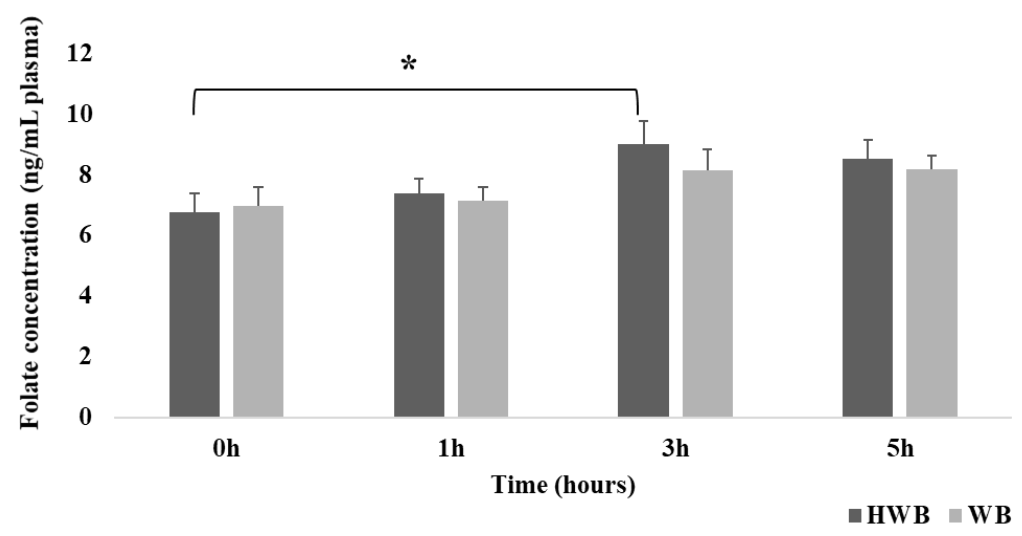

Figure 3 Plasma folate (mean $\pm S E M$, for $n=8$ volunteers) for at baseline $(0 \mathrm{~h}$ ), and 1,3 , $5 \mathrm{~h}$ after consumption of high wheat bran (HWB, black) and recommended serving of wheat bran (WB, grey). Where * stands for $p<0.05$ for significant change in time versus baseline $(0 \mathrm{~h})$, when calculated with t-test.

\subsection{Effect of SCFA on Cell Viability and Determination of Anti-inflammatory Activity}

The cells were found to be between 94 and $100 \%$ viable without stimulation and between 90 and $93 \%$ viable when stimulated with IL-1 $1 \beta$. When unstimulated the SCFA-treated fibroblast colon cells produced a significantly $(p<0.01)$ lower amount of prostanoids than the untreated control $(10.54 \pm 1.74 \mathrm{ng})$ only in the presence of acetate (Figure 4). In the presence of an inflammation stimulator (IL-1 $\beta$ ), all of the SCFA significantly reduced the amount of prostanoids produced. In the presence of acetate $(24.69 \pm 1.98 \mathrm{ng}, \mathrm{p}<0.001$, t-test), propionate $(26.46 \pm 2.95 \mathrm{ng}, \mathrm{p}<0.01$, t-test), butyrate $(21.92 \pm 3.73 \mathrm{ng}, \mathrm{p}<0.001$, t-test), and in presence of their mixture was the lowest concentration of the prostanoids produced (20.69 $\pm 1.03 \mathrm{ng}, \mathrm{p}<0.001$, t-test). 


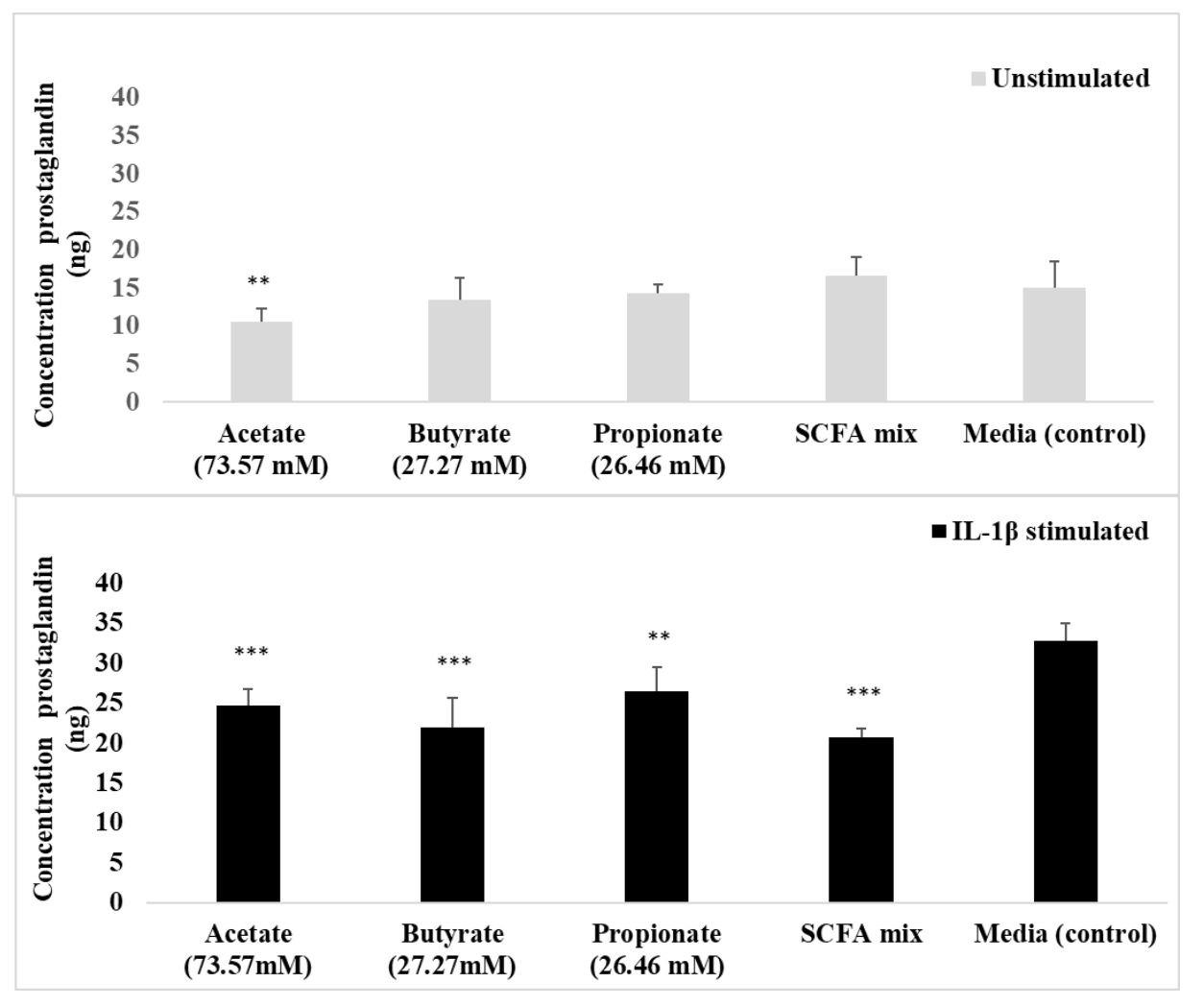

Figure 4 Prostanoid concentrations (mean \pm STD, $n=6$ ) in human colonic fibroblast cells (CCD-18Co) with (black) and without (grey) cytokine-induced stimulation with IL-1 $\beta$ in the presence of short chain fatty acids (SCFAs). SCFAs were tested at mean in vivo concentrations; $73.57 \mathrm{mM}, 27.27 \mathrm{mM}$ and $24.26 \mathrm{mM}$ for acetate, butyrate and propionate respectively. The mixture was a combination of all three SCFAs at the relevant concentrations. The non-supplemented media was used as control. Where * stands for $p<0.05, * *$ for $p<0.01$ and $* * *$ for $p<0.001$, for significant change(t-test). The significance refers to the difference in prostanoid concentration between media control and SCFAs presence in both cases with or without stimulation for inflammation.

\section{Discussion}

After the consumption of one recommended serving of wheat bran cereal (WB; $40 \mathrm{~g}$ ), the increase in concentration of the three major SCFAs in faecal samples (compared with the baseline) was higher than after the consumption of three times the recommended serving of wheat bran cereal (HWB; $120 \mathrm{~g}$ ) and in particular resulted in a $62.19 \%$ increase vs a $2.3 \%$ decrease in faecal butyrate levels as a proportion of the total SCFAs following intakes of the standard WB and HWB servings respectively. This suggests that increasing cereal fibre intakes above the recommended portion size does not translate into greater levels of faecal SCFAs. One possible explanation is that markedly increasing the portion size could impact on gut transit, with the high level of fibre increasing faecal bulking and gut transit and resulting in lower bacterial fermentation of dietary fibre and an increase in the presence of unfermented dietary fibre in stool samples [19, 20]. However, this is only a hypothetical explanation as unfermented dietary fibres in the stool were not measured in this study. This may therefore provide evidence for why the recommended portion size of bran may actually provide more benefits, with respect to faecal butyrate levels, than the high 
serving. Therefore, increasing the amount of cereal consumed at one meal (i. e. consuming all the daily recommended dietary amount of $30 \mathrm{~g}$ ) seems unlikely to provide additional benefits with respect to SCFA levels in faecal samples to promote gut health. In this case $40 \mathrm{~g}$ of wheat bran cereals, equivalent to $11 \mathrm{~g}$ of dietary fibre (one third of the daily recommended amount) in one meal would deliver potential benefits associated with SCFA production by allowing the fibre to be properly fermented and for the SCFAs (especially butyrate) to be produced and increased in the colon. Other studies have also reported on the impact of increasing dietary fibre intakes and found no significant increase in faecal SCFAs concentrations [21, 22]. The main difference between these studies and the present study is that in the present study the samples were collected following chronic consumption of a cereal fibre whilst in other studies the specific type of fibre (which was often mixed fibres present in fruits and vegetables, therefore likely to be rich in pectin) and source is not specified.

There is a plethora of evidence of the benefits associated with consumption of high and recommended amounts of dietary fibre in diets, such as increase gut motility [23], body weight control [24], decreased chronic inflammation [25], and a reduction in cardiovascular disease [26], however details on the source and the quantity of fibre consumed is sometimes lacking.

The folate levels were elevated, but only significantly different from baseline following the consumption of the HWB meal, and not after the WB serving; demonstrating that the folate in the bran cereals is likely to be bioavailable in a concentration dependant manner. This is important as achieving and maintaining a clinical normal range of folate in blood through diet is a public health priority due to the multiple implications and role of folate in human health. This also proves that the whole meal type foods such all-bran cereals are a valuable source of bioavailable folate to complement the mandatory UK fortification of foods [7].

It is essential to identify the types of cells, immune responses, tissue inflammation, and diseases that are highly responsive to SCFA-based therapies or high dietary fibre consumption. Although upregulated PGHS-2 and increased prostanoid synthesis are implicated in the early stages of colorectal neoplasia, its expression is hardly detectable in normal and pre-malignant colorectal epithelium and is perhaps confined to sub-epithelial cells, such as fibroblasts [27]. The colonic fibroblasts are potentially important targets, as cytokine induction results in a significant increase of PGHS-2 and that fibroblasts and their products are crucial determinants of epithelial cell behaviour; research suggesting [28] that fibroblasts could be important cytokine targets in the colon. Therefore, the determination of anti-inflammatory activity using a previously validated fibroblast cell model [15] and is important this will give important information of the role of SCFAs in inflammatory processes. Research has also shown that circulating SCFA can have an effect on systemic macrophage and dendritic cell (DC) biology, illustrating the strong connection between dietary fibre intake and many types of immune responses under the control of DC or macrophages [29]. Consequently, is it important to determine the nutritionally relevant concentration of both SCFA at both the systemic and gut levels achieved after fibre-rich meal consumption by humans.

It was observed that unstimulated fibroblast colon cells produced a significantly lower amount of prostanoids than the untreated control cells, only in the presence of acetate. This is important, since acetate was present at the highest levels in the faecal water samples and suggests a role in maintaining gut homeostasis. All SCFAs at the nutritionally relevant concentrations measured in volunteers' faecal samples, significantly reduced the production of neoplastic prostanoids when the cells were stimulated with II-1 $\beta$, with the SCFA mixture having the strongest effect. This suggests 
that SCFAs will play a role in protecting against upregulation of inflammation. Another key and important finding of this paper is that butyric acid, which had the overall highest increase following consumption of wheat bran cereals gave the highest anti-inflammatory response.

\section{Study Limitations}

The present study has several limitations. A relatively small sample size of eight participants limits the conclusions that can be drawn from the study, highlighting the importance to replicate our findings in a larger sample size (especially on the trends observed for plasma folate distribution). Additionally, the sequence by which the two portion sizes of cereal were consumed in was not randomized, however the timing of the interventions was adequately spaced out (minimum two weeks between each) and therefore there should be no carry over between treatments. Faecal SCFA levels are a proxy for intestinal levels however because as much (around 95\%) of the acids are likely to be rapidly absorbed by the colonocytes [30].

\section{Conclusions}

This study suggests that consumption of a recommended serving of wheat bran breakfast cereal of $40 \mathrm{~g}$, representing $11 \mathrm{~g}$ dietary fibre in one meal, is sufficient to support a butyrogenic fermentation with both butyrate levels and proportions increased compared to baseline samples. This is important for gut heath as butyrate is the main energy source for colonocytes [31, 32]. Wheat bran breakfast cereals are an important dietary source of fibre and the recommended $40 \mathrm{~g}$ serving could provide approximate $11 \mathrm{~g}$ of the daily recommendation for fibre. The study also suggests that meals with a higher fibre content, such as consuming all the daily recommended fibre intake in one meal may not necessarily be beneficial, as the promotion of faecal bulking is likely to increase gut transit and potentially decrease microbial fermentation.

Nutritionally relevant concentrations of SCFAs are likely to significantly inhibit colonic inflammation by reducing the formation of prostanoids; moreover, a mixture of the measured SCFAs proved to be the most efficient. Furthermore, acetate-treated fibroblasts produced a significantly lower endogenous amount of prostanoids compared to the control suggesting a role in gut homeostasis. In addition, wheat bran-based foods (breakfast cereals) are likely to contribute to maintaining clinically normal ranges of plasma folates.

\section{Acknowledgments}

The authors are grateful to all the volunteers for their participance in this human study. The authors also are thankful for the assistance from Karen Taylor and Jean Bryce for the preparation of the study diets and Sylvia Stephen, David Bremner and Lorna Hermitage for their support in the Human Nutrition Unit.

\section{Additional Materials}

The following additional materials are uploaded the page of this paper.

1. Table S1: Actual faecal sample weights (g) produced by the volunteers at baseline and post intervention following HWB and WB. 


\section{Author Contributions}

The authors' responsibilities are as follows: WRR was responsible for the study concept and design; MN and WRR were responsible for the data collection and collation, MN, SEA, SHD, SD, PV, NJV, GH and WRR were responsible for the samples and data analysis, MN, SHD, WRR, SD and TH were responsible for the first draft and critical revision of the manuscript for important intellectual content.

\section{Funding}

We acknowledge financial support from Kellogg Europe and The Scottish Government's Rural and Environment Science and Analytical Services (RESAS) division.

\section{Competing Interests}

Kellogg Europe contributed with financial support to the human dietary intervention study. The authors declare that there are no other conflicts of interest.

\section{References}

1. GOV.UK. The Scientific Advisory Committee on Nutrition recommendations on carbohydrates, including sugars and fibre from carbohydrates and health report. London: Scientific Advisory Committee on Nutrition; 2015.

2. Hooper B, Spiro A, Stanner S. $30 \mathrm{~g}$ of fibre a day: An achievable recommendation? Nutr Bull. 2015; 40: 118-129.

3. Hoare J, Henderson L, Bates C, Swan G. The National Diet and Nutrition Survey (NDNS): Adults age 19-64. London: Palgrave Macmillan; 2004.

4. Stevenson LE, Phillips F, O'Sullivan K, Walton J. Wheat bran: Its composition and benefits to health, a European perspective. Int J Food Sci Nutr. 2012; 63: 1001-1013.

5. Shewry P. The Healthgrain programme opens new opportunities for improving wheat for nutrition and health. Nutr Bull. 2009; 34: 225-231.

6. The Scientific Advisory Committee on Nutrition. Folate and disease prevention. London: The Stationery Office; 2006.

7. GOV.UK. Proposal to add folic acid to flour: Consultation response [Internet]. London: GOV.UK; 2021. Available from: https://www.gov.uk/government/consultations/adding-folic-acid-toflour/outcome/proposal-to-add-folic-acid-to-flour-consultation-response.

8. Bailey LB, Stover PJ, McNulty H, Fenech MF, Gregory III JF, Mills JL, et al. Biomarkers of nutrition for development-folate review. J Nutr. 2015; 145: 1636S-1680S.

9. The Scientific Advisory Committee on Nutrition. Folic acid and colorectal cancer risk: Review of recommendation for mandatory folic acid fortification [Internet]. London: Scientific Advisory Committee on Nutrition; 2009. Available from: https://assets.publishing.service.gov.uk/government/uploads/system/uploads/attachment_d ata/file/339293/SACN Report to CMO on folic acid and colorectal cancer risk .pdf.

10. Scott KP, Duncan SH, Flint HJ. Dietary fibre and the gut microbiota. Nutr Bull. 2008; 33: 201-211.

11. Scott KP, Gratz SW, Sheridan PO, Flint HJ, Duncan SH. The influence of diet on the gut microbiota. Pharmacol Res. 2013; 69: 52-60. 
12. Cummings J, Pomare EW, Branch WJ, Naylor CP, MacFarlane G. Short chain fatty acids in human large intestine, portal, hepatic and venous blood. Gut. 1987; 28: 1221-1227.

13. Klepinina L, Klepinin A, Truu L, Chekulayev V, Vija H, Kuus K, et al. Colon cancer cell differentiation by sodium butyrate modulates metabolic plasticity of Caco-2 cells via alteration of phosphotransfer network. PloS ONE. 2021; 16: e0245348.

14. Flint HJ, Scott KP, Louis $\mathrm{P}$, Duncan SH. The role of the gut microbiota in nutrition and health. Nat Rev Gastroenterol Hepatol. 2012; 9: 577-589.

15. Russell WR, Drew JE, Scobbie L, Duthie GG. Inhibition of cytokine-induced prostanoid biogenesis by phytochemicals in human colonic fibroblasts. Biochim Biophys Acta Mol Basis Dis. 2006; 1762: 124-130.

16. Neacsu M, McMonagle J, Fletcher RJ, Hulshof T, Duncan SH, Scobbie L, et al. Availability and dose response of phytophenols from a wheat bran rich cereal product in healthy human volunteers. Mol Nutr Food Res. 2017; 61: 1600202.

17. Belenguer A, Duncan SH, Calder AG, Holtrop G, Louis P, Lobley GE, et al. Two routes of metabolic cross-feeding between Bifidobacterium adolescentis and butyrate-producing anaerobes from the human gut. Appl Environ Microbiol. 2006; 72: 3593-3599.

18. Duncan SH, Russell WR, Quartieri A, Rossi M, Parkhill J, Walker AW, et al. Wheat bran promotes enrichment within the human colonic microbiota of butyrate-producing bacteria that release ferulic acid. Environ Microbiol. 2016; 18: 2214-2225.

19. Flint HJ, Duncan SH, Louis P. The impact of nutrition on intestinal bacterial communities. Curr Opin Microbiol. 2017; 38: 59-65.

20. Müller M, Canfora EE, Blaak EE. Gastrointestinal transit time, glucose homeostasis and metabolic health: Modulation by dietary fibers. Nutrients. 2018; 10: 275.

21. Yamamura R, Nakamura K, Kitada N, Aizawa T, Shimizu Y, Nakamura K, et al. Associations of gut microbiota, dietary intake, and serum short-chain fatty acids with fecal short-chain fatty acids. Biosci Microbiota Food Health. 2020; 39: 11-17.

22. Oliver A, Chase AB, Weihe C, Orchanian SB, Riedel SF, Hendrickson CL, et al. High-fiber, wholefood dietary intervention alters the human gut microbiome but not fecal short-chain fatty acids. Msystems. 2021; 6: e00115-21.

23. Rao SS, Yu S, Fedewa A. Systematic review: Dietary fibre and FODMAP-restricted diet in the management of constipation and irritable bowel syndrome. Aliment Pharmacol Ther. 2015; 41: 1256-1270.

24. Solah VA, Kerr DA, Hunt WJ, Johnson SK, Boushey CJ, Delp EJ, et al. Effect of fibre supplementation on body weight and composition, frequency of eating and dietary choice in overweight individuals. Nutrients. 2017; 9: 149.

25. Kabisch S, Meyer NM, Honsek C, Gerbracht C, Dambeck U, Kemper M, et al. Obesity does not modulate the glycometabolic benefit of insoluble cereal fibre in subjects with prediabetes-a stratified post hoc analysis of the optimal fibre trial (OptiFiT). Nutrients. 2019; 11: 2726.

26. Kim Y, Je Y. Dietary fibre intake and mortality from cardiovascular disease and all cancers: $A$ meta-analysis of prospective cohort studies. Arch Cardiovasc Dis. 2016; 109: 39-54.

27. Zhu Y, Hua P, Lance P. Cyclooxygenase-2 expression and prostanoid biogenesis reflect clinical phenotype in human colorectal fibroblast strains. Cancer Res. 2003; 63: 522-526.

28. Kim EC, Zhu Y, Andersen V, Sciaky D, Cao HJ, Meekins H, et al. Cytokine-mediated $\mathrm{PGE}_{2}$ expression in human colonic fibroblasts. Am J Physiol Cell Physiol. 1998; 275: C988-C994. 
29. Thorburn AN, Macia L, Mackay CR. Diet, metabolites, and "western-lifestyle" inflammatory diseases. Immunity. 2014; 40: 833-842.

30. Den Besten G, Van Eunen K, Groen AK, Venema K, Reijngoud DJ, Bakker BM. The role of shortchain fatty acids in the interplay between diet, gut microbiota, and host energy metabolism. J Lipid Res. 2013; 54: 2325-2240.

31. Hamer HM, Jonkers DM, Venema K, Vanhoutvin SA, Troost FJ, Brummer RJ. The role of butyrate on colonic function. Aliment Pharmacol Ther. 2008; 27: 104-119.

32. Louis P, Hold GL, Flint HJ. The gut microbiota, bacterial metabolites and colorectal cancer. Nat Rev Microbiol. 2014; 12: 661-672.

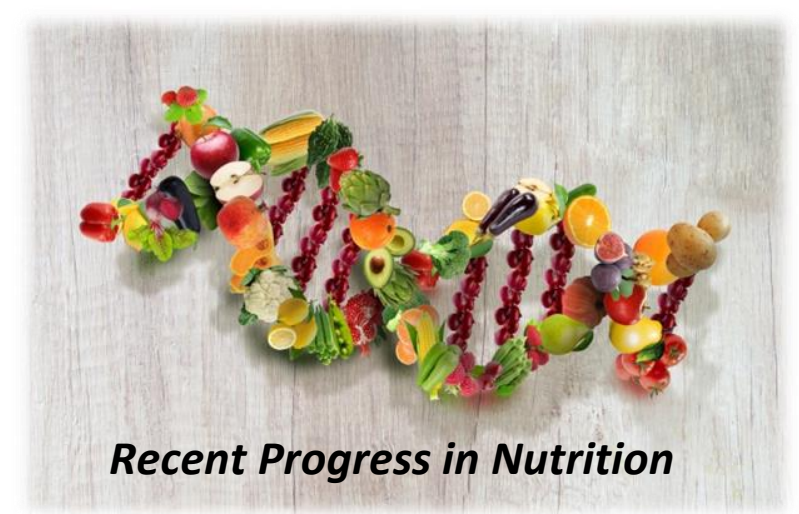

Enjoy Recent Progress in Nutrition by:

1. Submitting a manuscript

2. Joining in volunteer reviewer bank

3. Joining Editorial Board

4. Guest editing a special issue

For more details, please visit:

http://www.lidsen.com/journals/rpn 